\section{Commentary: Are we at a CROSSroads in neoadjuvant therapy for esophageal cancer?}

\author{
Stephen R. Broderick, MD, MPHS
}

It has been 12 years since publication of the Chemoradiotherapy for Oesophageal Cancer followed by Surgery Study trial set the standard of care for neoadjuvant chemoradiotherapy followed by esophagectomy for locally advanced esophageal cancer. ${ }^{1}$ More recently, the CALGB 80803 trial demonstrated higher rates of pathologic complete response with a fluorouracil-based, positron-emission tomographydirected neoadjuvant strategy. ${ }^{2}$ Long-term outcomes from this trial are pending. Recurrence, particularly distant recurrence, continues to limit survival in locally advanced esophageal cancer. Two analyses of patterns of recurrence after curative resection of esophageal cancer have been performed by Memorial Sloan Kettering Cancer Center. In 2013 , Lou and colleagues ${ }^{3}$ demonstrated that $72 \%$ of recurrences included distant metastases. Similarly, in 2019, Nobel and colleagues ${ }^{4}$ showed that even among patients with pathologic T1-2N0 disease recurrence was distant in 56\% of patients. These distant failures highlight the need for more effective systemic therapy in esophageal cancer.

Immune checkpoint inhibitors (ICIs) have become important therapeutic agents in many cancers. Evaluation of ICIs in patients with metastatic esophageal cancer have been promising in subsets of patients..$^{5}$ Recently, early data from the ongoing KEYNOTE-590 (NCT03189719) and CheckMate-577 (NCT02743494) trials presented at the European Society for Medical Oncology 2020 Virtual Congress suggest favorable results in both the advanced at adjuvant settings. It is rational to evaluate these promising systemic therapies in the neoadjuvant setting given the frequency of systemic recurrences after esophageal resection.

\footnotetext{
From the Division of Thoracic Surgery, Department of Surgery, Johns Hopkins Medical Institutions, Baltimore, Md.

Disclosures: Dr Broderick has received consulting fees from Bristol Myers Squibb and AstraZeneca. He also serves on the speakers bureau for AstraZeneca.

The Journal policy requires editors and reviewers to disclose conflicts of interest and to decline handling or reviewing manuscripts for which they may have a conflict of interest. The editors and reviewers of this article have no conflicts of interest.

Received for publication Dec 22, 2020; revisions received Dec 22, 2020; accepted for publication Dec 23, 2020; available ahead of print Dec 31, 2020.

Address for reprints: Stephen R. Broderick, MD, MPHS, Division of Thoracic Surgery, Department of Surgery, Johns Hopkins Medical Institutions, $600 \mathrm{~N}$ Wolfe St, Blalock 240, Baltimore, MD 21287 (E-mail: sbroder7@jhmi.edu).

J Thorac Cardiovasc Surg 2023;165:e87-8 $0022-5223 / \$ 36.00$

Copyright (c) 2020 Published by Elsevier Inc. on behalf of The American Association for Thoracic Surgery

https://doi.org/10.1016/j.jtcvs.2020.12.107
}

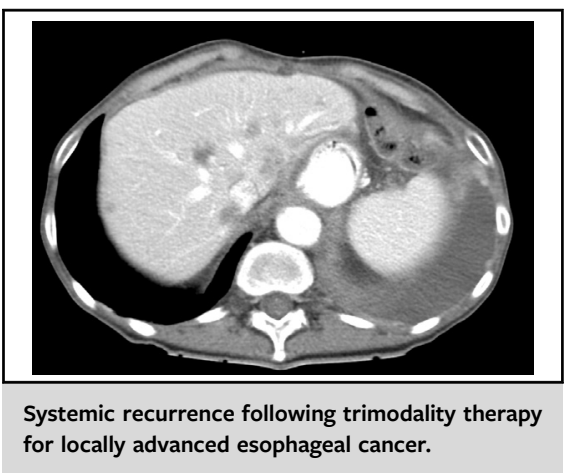

CENTRAL MESSAGE

Improved systemic control is needed in esophageal cancer. This study suggests safety and feasibility of esophagectomy following combined neadjuvant therapy with durvalumab and chemoradiotherapy.

In this retrospective analysis by Sihag and colleagues, ${ }^{6}$ the group from Memorial Sloan Kettering Cancer Center report on the safety and feasibility of esophagectomy following the combination of immunotherapy and chemoradiation. Patients in the study received 2 doses of durvalumab before chemoradiotherapy and esophagectomy. Appropriately, the authors have limited their analysis to short-term perioperative outcomes, delaying discussion of response until trial completion and further analysis. Immune-related toxicities occurred in $28 \%$ of patients, but did not result in delays to surgery. There were no episodes of pneumonitis. The crude rates of perioperative morbidities were similar to those seen in most large series and database analyses. The authors selected a comparison group from their institutional database and found only minor differences in outcome between the study and control groups after multivariable analysis. This analysis is statistically limited by the small study group and relatively few adverse events. In addition, the study group has a very low rate of smoking history or comorbid pulmonary disease. Nonetheless, the descriptive statistics regarding completion of therapy, lack of delays to resection, and absence of adverse safety signals are important contributions to the field.

This initial report of perioperative outcomes following neoadjuvant therapy with combined ICI and 
chemoradiotherapy does not suggest significant untoward effects related to the addition of immunotherapy to current trimodality regimens. As we continue to employ novel agents in the neoadjuvant setting, it is incumbent upon surgeon investigators to report clinical results. Hopefully, as this and other esophageal trials reach completion we will see evidence of response to therapy and ultimately in improvement in survival.

\section{References}

1. van Hagen $\mathrm{P}$, Hulshof MC, van Lanshot JJ, Steyerberg EW, van Berge Henegouwen MI, Wijnhoven BP, et al. Preoperative chemoradiotherapy for esophageal and junctional cancer. N Engl J Med. 2012;366:2074-84.
2. Goodman KA, Hall N, Bekaii-Saab Ts, Ou F-S, Twohy E, Meyers MO, et al. Survival outcomes from CALGB 80803 (Alliance): a randomized phase II trial of PET scan-directed combined modality therapy for esophageal cancer. J Clin Oncol. 2018;36:4012.

3. Lou F, Sima C, Adusumilli P, Bains M, Sarkaria I, Rusch VW, et al. Esophageal cancer recurrence patterns and implications for surveillance. J Thorac Oncol. 2013;8:1558-62.

4. Nobel T, Livschitz J, Xing X, Barbetta A, Hsu M, Tan KS, et al. Surveillance implications of recurrence patterns in early node-negative esophageal adenocarcinoma. Ann Thorac Surg. 2019;108:1640-7.

5. Janjigian YY, Bendell J, Calvo E, Kim JW, Ascerito PA, Sharma P, et al. CheckMate-032 study: efficacy and safety of nivolumab and nivolumab plus ipilimumab in patients with metastatic esophagogastric cancer. J Clin Oncol. 2018;36:2836-44.

6. Sihag S, Ku GY, Tan KS, Nussenzweig S, Wu A, et al. Safety and feasibility of esophagectomy following combined immunotherapy and chemoradiotherapy for esophageal cancer. J Thorac Cardiovasc Surg. 2021;161:836-43. 